\title{
Probiotics protect mice from CoCrMo particles-induced osteolysis
}

This article was published in the following Dove Press journal:

International Journal of Nanomedicine

27 July 2017

Number of times this article has been viewed

\section{Zhenheng Wang* \\ Kaiwen Xue* \\ Maosheng Bai \\ Zhantao Deng \\ Jingjing Gan \\ Gang Zhou \\ Hongbo Qian* \\ Nirong Bao \\ Jianning Zhao}

Department of Orthopaedics, Jinling Hospital, School of Medicine, Nanjing University, Nanjing, People's Republic of China

*These authors contributed equally to this work
Correspondence: Jianning Zhao;

Nirong Bao

Department of Orthopaedics, Jinling Hospital, School of Medicine, Nanjing University, 305 East Zhongshan

Road, Nanjing 210093, People's

Republic of China

Tel/fax +86 2580860015

Email zhaojianning.0207@I63.com;

bnrbnrn@sina.com

\begin{abstract}
Wear particle-induced inflammatory osteolysis is the primary cause of aseptic loosening, which is the most common reason for total hip arthroplasty (THA) failure in the med- and long term. Recent studies have suggested an important role of gut microbiota (GM) in modulating the host metabolism and immune system, leading to alterations in bone mass. Probiotic bacteria administered in adequate amounts can alter the composition of GM and confer health benefits to the host. Given the inflammatory osteolysis that occurs in wear debris-induced prosthesis loosening, we examined whether the probiotic Lactobacillus casei could reduce osteolysis in a mouse calvarial resorption model. In this study, L. casei markedly protected mice from CoCrMo particles (CoPs)-induced osteolysis. Osteoclast gene markers and the number of osteoclasts were significantly decreased in $L$. casei-treated mice. Probiotic treatment decreased the M1-like macrophage phenotype indicated by downregulation of tumor necrosis factor $\alpha$ (TNF- $\alpha$ ), interleukin (IL)- 6 and inducible nitric oxide synthase (iNOS) and increased the M2-like macrophage phenotype indicated by upregulation of IL-4, IL-10 and arginase. Collectively, these results indicated that the $L$. casei treatment modulated the immune status and suppressed wear particle-induced osteolysis in vivo. Thus, probiotic treatment may represent a potential preventive and therapeutic approach to reduced wear debris-induced osteolysis.

Keywords: wear particles, gut microbiota, nanotoxicity, macrophage polarization, inflammatory cytokines, aseptic loosening
\end{abstract}

\section{Introduction}

Total hip arthroplasty (THA) is one of the most effective treatments for various end-stage joint diseases. ${ }^{1-3}$ However, wear particle-induced osteolysis and the subsequent aseptic loosening remain the most common causes of arthroplasty failure and revision surgery in the med- to long term. ${ }^{4}$ Wear debris that is generated from implant components plays a key role in aseptic loosening. ${ }^{5}$ Wear particles are responsible for the activation of macrophages, which subsequently release various proinflammatory cytokines, such as tumor necrosis factor $\alpha$ (TNF- $\alpha$ ), interleukin (IL)-6 and IL-1 $\beta$. These inflammatory cytokines can increase osteoclast formation and activate osteoclast activity. The concept of macrophage polarization was recently considered to play a role in the pathological mechanism of aseptic loosening. ${ }^{1,6,7}$ M1 proinflammatory macrophages were activated in response to wear debris dominating the M2 antiinflammatory response. M1 macrophages secrete primarily proinflammatory cytokines such as TNF- $\alpha$, IL-6 and IL-1 $\beta$, while M2 macrophages produce anti-inflammatory mediators such as IL-4, IL-10 and IL-13. The increased ratio of M1/M2 macrophages from peri-implant tissues compared with osteoarthritic synovial tissues has been reported. ${ }^{7}$ Furthermore, the modulation of macrophage polarization was considered an effective means to mitigate wear particle-induced osteolysis. ${ }^{1,6}$ 
Recently, the gut microbiota (GM) has emerged as a potentially important regulator of systemic health. Perturbed GM composition has been postulated to be involved in a range of inflammatory conditions, within and outside the gut, including Crohn's disease, ulcerative colitis, multiple sclerosis, diabetes, rheumatoid arthritis, asthma and obesity. ${ }^{8}$ Sjogren et $\mathrm{al}^{9}$ have showed that the absence of GM in germ-free mice leads to increased bone mass. Probiotics are defined as live microorganisms whose administration in adequate amounts confers health benefits to the host by altering the composition of GM. ${ }^{8}$ Lactobacillus casei is usually used in dietary supplements, and previous studies have shown that $L$. casei possesses strong anti-inflammatory activity. ${ }^{10-14}$ In collagen-induced arthritis (CIA) animal model, oral administration of $L$. casei significantly decreased the serum levels of pro-inflammatory cytokines IL- 6 and TNF- $\alpha$ and increased the levels of IL-10. ${ }^{13}$ In another study, administration of $L$. case $i$ suppressed clinical symptoms in experimental rheumatoid arthritis, including paw swelling, lymphocyte infiltration and destruction of cartilage tissues, and the therapeutic efficacy was associated with an increase in anti-inflammatory cytokines while decreasing proinflammatory cytokines. ${ }^{12}$ Wear-debris activated macrophages, which released an array of proinflammatory cytokines, resulted in the recruitment, differentiation and maturation of osteoclast precursors. ${ }^{15}$ Given the important role of macrophages in the pathological mechanisms of aseptic loosening and the key role of GM in systemic bone health and inflammatory conditions, questions have been raised as to probiotic supplements affect wear debris-induced local inflammatory conditions and osteolysis in the pathological process of aseptic loosening.

In this study, we examined the effect of a probiotic (L. casei) in a wear particles-treated mice calvarial resorption model. Interestingly, L. casei attenuated CoCrMo particles (CoPs)-induced osteolysis and osteoclast formation.
Further, our results indicated that these effects may be due to the decrease in M1-like macrophages and the increase in M2-like macrophages in local tissue. Thus, the administration of probiotics may be a potential therapeutic approach for the treatment of aseptic loosening.

\section{Materials and methods \\ Bacterial culture}

Lactobacillus casei (ATCC 334) was purchased from the American Type Culture Collection. Lactobacillus casei was cultured under anaerobic conditions in de Mann Rogosa Sharpe Agar (MRS) at $37^{\circ} \mathrm{C}$.

\section{Particle preparation}

The characteristics of the CoPs are described in Figure 1. The particles were autoclaved for 15 minutes at $121^{\circ} \mathrm{C}$ and $15 \mathrm{psi}$ for sterilization and then suspended in phosphate-buffered saline (PBS). The particles were endotoxin-free, as determined by a commercial detection kit (chromogenic end-point TAL with diazo coupling kit; Xiamen Houshiji, Ltd, Xiamen, People's Republic of China). To obtain essential information on the size and shape of the nanoparticles, transmission electron microscopy (TEM) was carried out. A few drops of deionized water-dispersed particles were cast on a 300-mesh carbon-coated copper grid. TEM images of the sample were collected using a transmission electron microscope (JEOL Ltd., Tokyo, Japan). From the acquired TEM images, particle sizes were measured by manually measuring the particle diameters using ImageJ software as previously described. ${ }^{16}$

\section{In vivo calvarial resorption model and probiotic treatment}

The mice were obtained from the experimental animal center of Jinling Hospital (Nanjing, People's Republic of China),

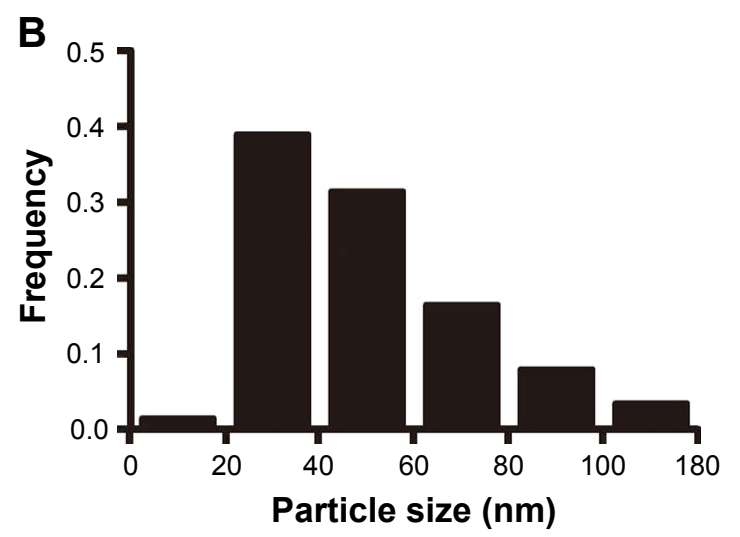

Figure I Characterizations of CoPs.

Notes: (A) Representative TEM images of CoPs. (B) CoPs size distribution. (A) Scale bar $100 \mathrm{~nm}$. (B) Particles with sizes of $52.2 \pm 27.5$ (mean \pm SD).

Abbreviations: CoPs, CoCrMo particles; TEM, transmission electron microscopy; SD, standard deviation. 
and the research was approved by the Nanjing Jinling Hospital Ethics Committee. All animals received humane care in accordance with Chinese legal requirements (the Laboratory Animal Management Regulations [January 8, 2011, revision]). Animals were divided into three groups: group I, sham-operated controls; group II, CoPs treatment, and group III, CoPs plus L. casei. In group III, the animals were treated with $2 \times 10^{8} \mathrm{CFU} / \mathrm{mL}$ (colony-forming units $/ \mathrm{mL}$ ) of $L$. case $i$ suspended in $500 \mu \mathrm{L}$ distilled water administered directly into the stomach with a gavage needle three times per week for 8 weeks. In group II, the animals received $500 \mu \mathrm{L}$ distilled water. The wear particle-induced calvarial osteolysis model in 8-week-old C57BL/J6 mice has been described previously. ${ }^{17,18}$ Briefly, the mice were anesthetized, and the cranial periosteum was separated from the calvarium by sharp dissection. Forty microliters $(40 \mathrm{mg} / \mathrm{mL})$ of the CoPs suspension was embedded under the periosteum around the middle suture of the calvaria. Group I mice received $40 \mu \mathrm{L}$ PBS only (sham group). Group II and group III mice were administered with distilled water or bacterial for another 2 weeks after surgery. Then, the animals were sacrificed, and the calvarial caps were removed by dissecting the bone free from the underlying brain tissue.

\section{Calvaria culture}

As previously described, ${ }^{6}$ each of the calvaria was placed into a well of a 12-well plate and cultured with $2 \mathrm{~mL}$ Dulbecco's Modified Eagle's Medium (DMEM; Thermo Fisher Scientific, Waltham, MA, USA) for 24 hours at $37^{\circ} \mathrm{C}$ with $5 \% \mathrm{CO}_{2}$. The culture medium was collected and stored at $-20^{\circ} \mathrm{C}$ for assay of receptor activator of nuclear factor (NF)- $\kappa B$ ligand (RANKL), osteoprotegerin (OPG), TNF- $\alpha$, IL-6, IL-4 and IL-10.

\section{Micro-computed tomography (micro-CT) scanning}

After culturing in DMEM, the mouse calvaria were analyzed by a high-resolution micro-CT (SkyScan1176; SkyScan, Aartselaar, Belgium) at a resolution of $18 \mu \mathrm{m}$ and X-ray energy settings of $45 \mathrm{kV}$ and $550 \mu \mathrm{A}$. A square region of interest (ROI) around the midline suture was selected for further qualitative and quantitative analysis after reconstruction. Bone volume/total volume (BV/TV) and the percentage of total porosity within the ROI were measured with the software provided with the micro-CT system, as reported previously. ${ }^{19}$

\section{Real-time polymerase chain reaction (PCR)}

Total RNA from the calvarial bone was prepared using TRIzol reagent (Thermo Fisher Scientific) as previously described. ${ }^{18}$ Real-time PCR was performed according to the manufacturer's protocols. Primers for $\beta$-actin were used as internal controls. The primers used in this study are listed in Table 1.

\section{Histologic and histomorphometric analysis}

After micro-CT analysis, a portion of the calvarias were decalcified in 15\% ethylene diamine tetraacetic acid (EDTA)-PBS for 3 weeks and embedded in paraffin. The sections were stained with hematoxylin and eosin (HE) and tartrateresistant acidic phosphatase (TRAP; Sigma-Aldrich, 386A) by standard methods. The specimens were then observed and photographed with a light microscope. The number of osteoclasts counted in each sample was reported as previously described. ${ }^{20}$

\section{Enzyme-linked immunosorbent assay (ELISA) detection}

The RANKL, OPG, TNF- $\alpha$, IL-6, IL-4 and IL-10 levels were quantified using ELISA kits (Jinyibai Biological Technology Co. Ltd, Nanjing, People's Republic of China). All procedures were performed according to the manufacturer's instructions.

\section{Statistical analysis}

The results were expressed as the mean \pm standard error of the mean (SEM). The differences among groups were analyzed by analysis of variance (ANOVA), followed by Dunnett's test or Bonferroni's post hoc test. Additionally, comparisons between two groups were analyzed using Student's $t$-tests. $P$-value $<0.05$ was considered significantly different.

Table I The primers for RT-PCR assay in this study

\begin{tabular}{ll}
\hline Primer name & Primer sequence (5'-3') \\
\hline ALP sense & GGACAGGACACACACACACA \\
ALP antisense & CAAACAGGAGAGCCACTTCA \\
OCN sense & CAGACACCATGAGGACCCTC \\
OCN antisense & GGGCGTGGCATCTGTGAGGT \\
TRAP sense & CTGGAGTGCACGATGCCAGCGACA \\
TRAP antisense & TCCGTGCTCGGCGATGGACCAGA \\
CTR sense & TGCAGACAACTCTTGGTTGG \\
CTR antisense & TCGGTTTCTTCTCCTCTGGA \\
c-fos sense & TCCAGATTGCTGGACAATGA \\
c-fos antisense & CCCGTCTTGGCATACATCTT \\
Cathepsin K sense & CTTCCAATACGTGCAGCAGA \\
Cathepsin K antisense & TCTTCAGGGCTTTCTCGTTC \\
$\beta$-actin sense & GGTGTGATGGTGGGAATGGG \\
$\beta$-actin antisense & ACGGTTGGCCTTAGGGTTCAG \\
\hline
\end{tabular}

Abbreviations: $R T$, reverse transcription; $P C R$, polymerase chain reaction; ALP, alkaline phosphatase; OCN, osteocalcin; TRAP, tartrate-resistant acidic phosphatase; CTR, calcitonin receptor. 


\section{Results}

\section{Characterizations of CoPs}

TEM was used to measure the morphology and size distribution of the CoPs. Most particles were round to oval-round shapes with some irregular morphology (Figure 1A). Eightyseven percent of the particles ranged from $20 \mathrm{~nm}$ to $80 \mathrm{~nm}$, with a mean diameter of $52.2 \mathrm{~nm}$ and a standard deviation of $27.5 \mathrm{~nm}$ (Figure 1B), which were similar with the wear debris retrieved from metal implants. ${ }^{21-25}$

\section{L. casei ameliorated CoPs-induced osteolysis}

To determine the effect of probiotic treatment on wear debris-induced osteolysis, 8-week-old mice were treated with
L. casei or vehicle for 10 weeks, starting 8 weeks before surgery (Figure 2A). CoPs-induced osteolysis was assessed by micro-CT with three-dimensional reconstruction. As shown in Figure 2B and Figure S1, the osteolysis induced by CoPs was suppressed by L. casei administration. Quantitative analysis of bone parameters confirmed that L. casei significantly increased the BV/TV and decreased the percentage of total porosity (Figure 2C and D). To further confirm the effect of $L$. casei on wear debris-induced bone loss, we processed histological and histomorphometric assessments. HE staining showed that sections in the sham group exhibited few osteolytic changes (Figure 2D). In the CoPstreated group, osteolysis had clearly occurred, whereas the L. casei-administered group exhibited decreased osteolysis

A

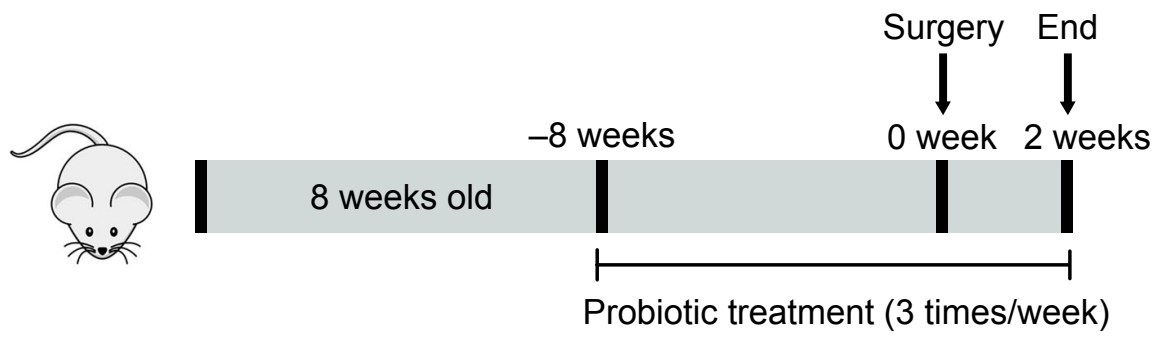

B

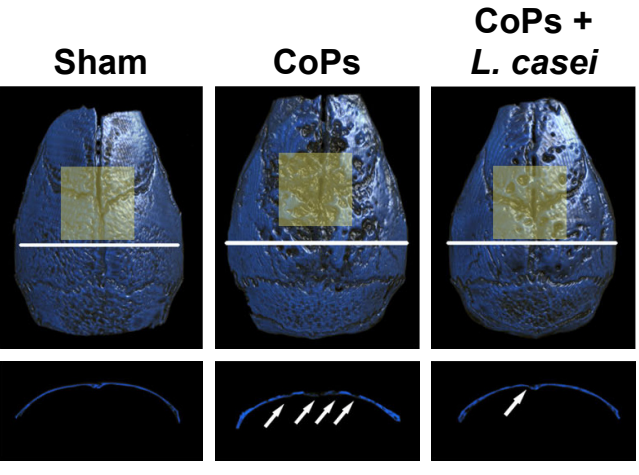

C
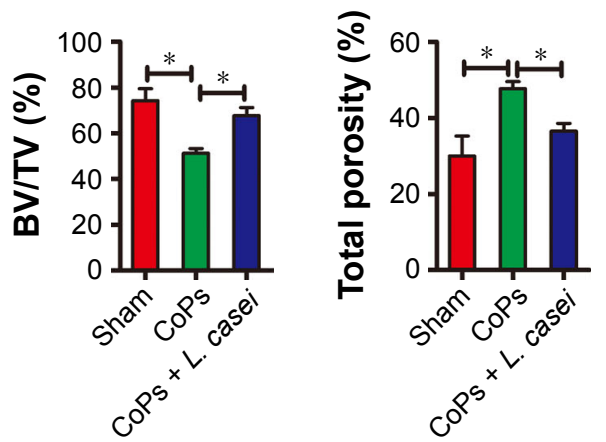

D

E
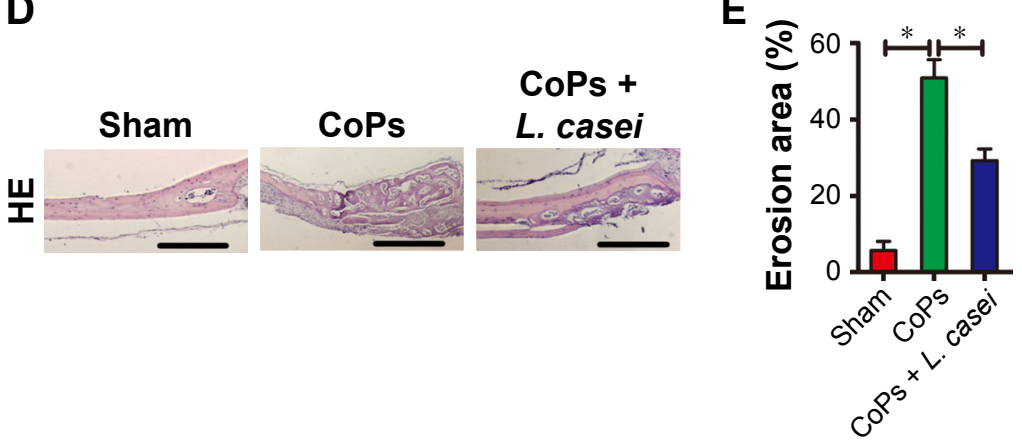

Figure 2 Lactobacillus casei ameliorated CoPs-induced mouse calvarial osteolysis.

Notes: (A) The 8-week-old mice were treated with either L. casei or vehicle preoperatively for 8 weeks ( 3 times/week) and postoperatively for 2 weeks (3 times/week). The animals were then sacrificed, and tissues were collected for later analysis. (B) Representative micro-CT with three-dimensional reconstructed images from each group. (C) BV/TV and the percentage of total porosity of each sample were measured. (D) Representative HE-stained images of calvaria from each group. (E) The erosion area (\%) of each group was measured in (D). The ROI in (B) was indicated by the yellow square box. The data are presented as the mean \pm SEM ( $=5-7$ ). $* P<0.05$. (D) Scale bar: $250 \mu \mathrm{m}$. Abbreviations: CoPs, CoCrMo particles; CT, computed tomography; BV/TV, bone volume/total volume; HE, hematoxylin and eosin; ROI, region of interest; SEM, standard error of the mean; L. casei, Lactobacillus casei. 
(Figure 2D). The erosion area induced by CoPs was reduced by $\sim 40 \%$ in the $L$. casei-treated group (Figure 2E). The data indicated that $L$. casei partly protected bone loss induced by CoPs.

\section{L. casei suppressed CoPs-induced osteoclast formation}

Normal bone metabolism is reliant on homeostasis between bone formation and degradation. Loss of bone stock in the peri-prosthetic area can be due to either the stimulation of bone resorption or the inhibition of bone formation. ${ }^{18,26,27}$ To explore the reason behind the protective effect of $L$. casei on bone stock, we first examined both osteoblast gene markers (alkaline phosphatase [ALP] and osteocalcin [OCN]) and osteoclast gene markers (TRAP and calcitonin receptor [CTR]). As shown in Figure 3A, osteoblast markers were markedly downregulated by CoPs. Although $L$. casei partly rescued the decrease in osteoblast markers, no significant differences were detected between the CoPs group and the CoPs and L. casei co-treatment group. In contrast, CoPs
B

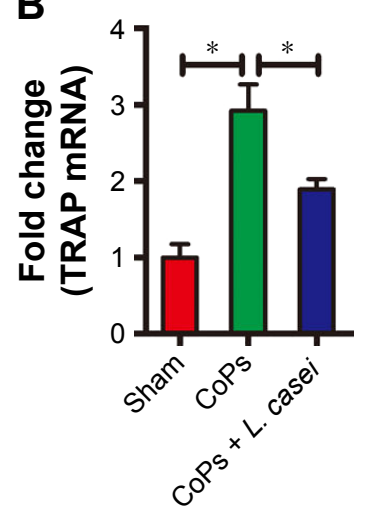

C

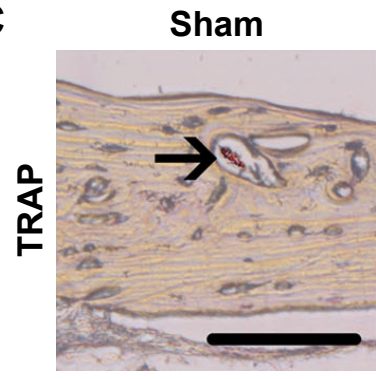

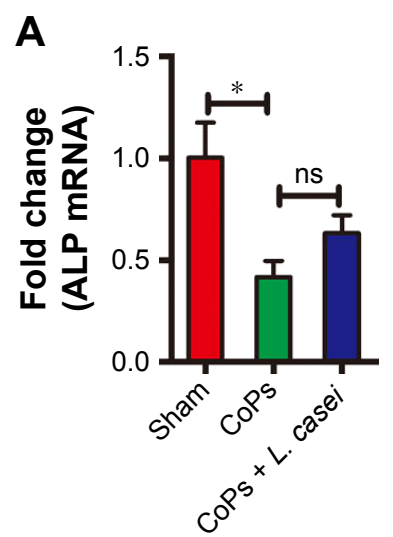

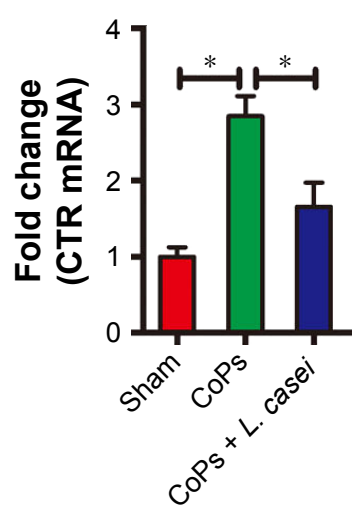

CoPs

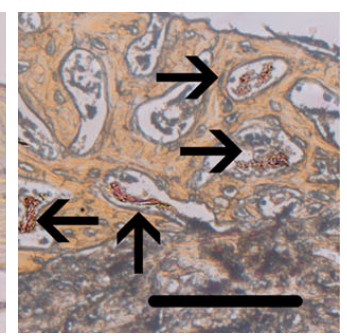

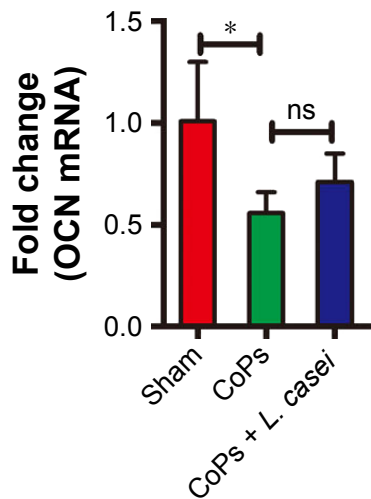
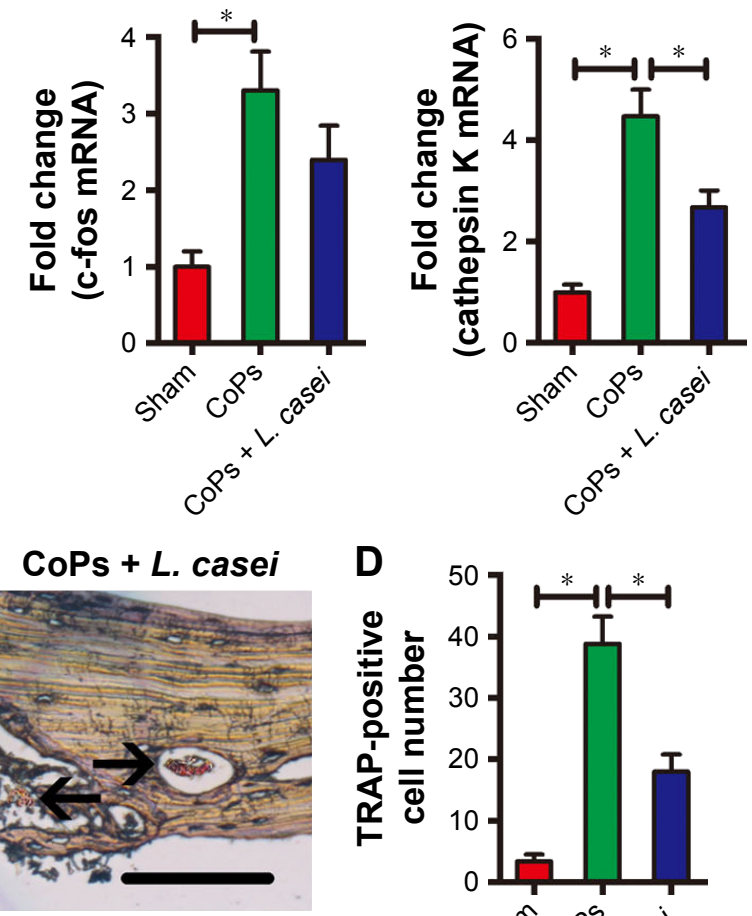

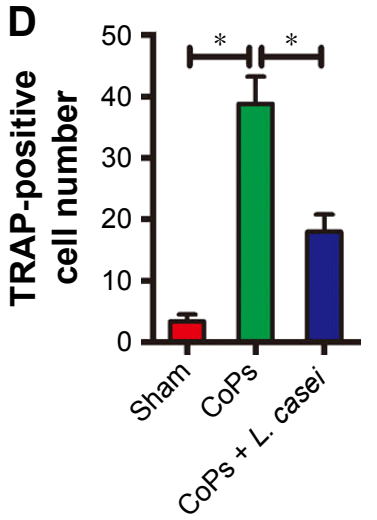

Figure 3 Lactobacillus casei inhibited CoPs-induced osteoclast formation.

Notes: (A) The levels of ALP and OCN mRNAs from each group were examined using real-time PCR. (B) The levels of TRAP, CTR, c-fos and cathepsin K mRNAs from each group were examined using real-time PCR. (C) Representative TRAP-stained images of calvaria from each group. Osteoclasts are indicated by arrows. (D) The number of TRAP-positive cells in each group was measured in (C). The data are presented as the mean \pm SEM $(n=5-7)$. ${ }^{*} P<0.05$. (C) Scale bar: $60 \mu \mathrm{m}$.

Abbreviations: CoPs, CoCrMo particles; ALP, alkaline phosphatase; OCN, osteocalcin; mRNA, messenger RNA; PCR, polymerase chain reaction; TRAP, tartrate-resistant acidic phosphatase; CTR, calcitonin receptor; SEM, standard error of the mean; ns, not significant; L. casei, Lactobacillus casei. 
treatment significantly increased the expression of osteoclast markers TRAP and CTR, and the upregulation was reversed by $L$. casei administration (Figure 3B). We also detected the other two osteoclast genes c-fos and cathepsin K. The results showed that the expression of cathepsin $\mathrm{K}$ messenger RNA (mRNA) induced by CoPs was inhibited by L. casei co-treatment (Figure 3B). The level of c-fos showed a similar tendency, although the data were not statistically significant (Figure 3B). In agreement with the earlier results, we detected a significant increase in TRAP-positive cells in CoPs-treated calvaria caps, which indicated the presence of osteoclasts (Figure 3C). CoPs treatment markedly increased the number of osteoclasts by 11-fold compared to the sham group (Figure 3D). Co-treatment with L. casei decreased the number of osteoclasts by $50 \%$ (Figure $3 \mathrm{C}$ and $\mathrm{D}$ ). These results suggested that the protective effect of $L$. casei on CoPs-induced osteolysis was due to the inhibition of osteoclastogenesis.

\section{L. casei did not affect RANKL and OPG expression in local tissue}

As RANKL and OPG play key roles in osteoclastogenesis and a previous study suggested that L. mix (containing Lactobacillus paracasei DSM 13434, Lactobacillus plantarum DSM 15312 and DSM 15313) treatment increased the expression of OPG in vivo, we explored whether $L$. casei regulated the levels of RANKL and OPG in local tissues. ${ }^{8,28}$ The calvaria were cultured in media for 24 hours, and then the culture media were collected for RANKL and OPG detection. As shown in Figure 4A and C, neither RANKL nor OPG in local tissues was modulated by $L$. casei.

\section{L. casei decreased the expression of MI markers and enhanced the expression of M2 markers in local tissue}

Inflammatory cytokines play key roles in wear debris-induced osteolysis and can modulate osteoclast formation and activity. ${ }^{2}$ Modulation of macrophages from the proinflammatory M1 to the anti-inflammatory M2 phenotype is considered as an important way to mitigate wear particle-induced osteolysis. ${ }^{1}$ We next investigated whether $L$. casei treatment affected the phenotypes of macrophages. The levels of M1 markers TNF- $\alpha$, IL-6 and iNOS in local tissue were upregulated in the CoPs-treated group compared with the sham group (Figure 5A). The upregulation was markedly attenuated by treatment with $L$. casei (Figure $5 \mathrm{~A}$ ). By contrast, the levels of M2 markers IL-4, IL-10 and arginase were decreased in the wear debris-treated group compared with the sham group (Figure 5B). After administration of L. casei, the levels of IL-4, IL-10 and arginase were significantly increased. In vitro, co-culture of human colonic mucosa with L. case $i$ significantly decreased the expression of TNF- $\alpha$ and IL-6, while increased the level of IL-4 (Figure S2 and Supplementary materials).

\section{Discussion}

Monocytes are versatile cells that can express different functional programs in response to microenvironmental signals. ${ }^{29}$ Fully polarized M1 and M2 macrophages represent extremes of a continuum of functional states. Most tissues contain highly specialized macrophage subsets. These tissue-resident macrophages, or M0 (undifferentiated) macrophages are
A

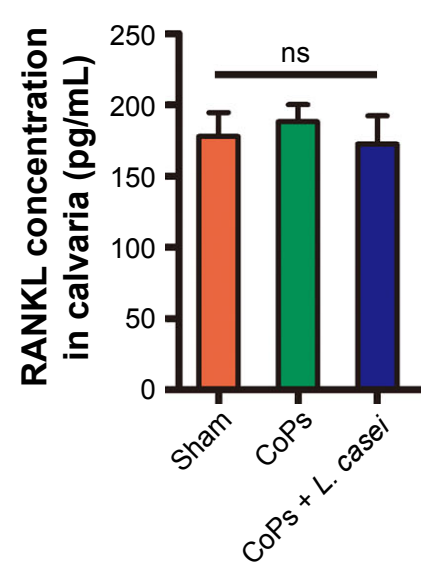

B

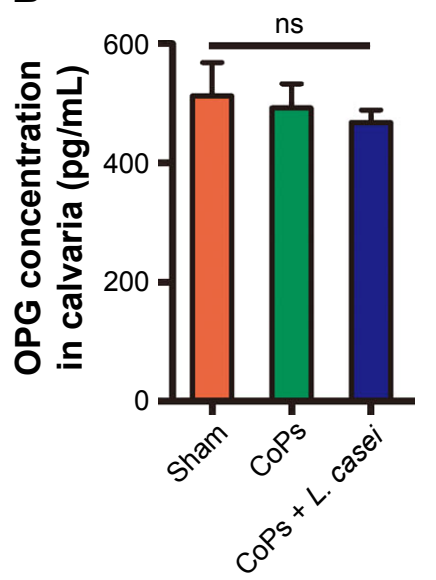

C

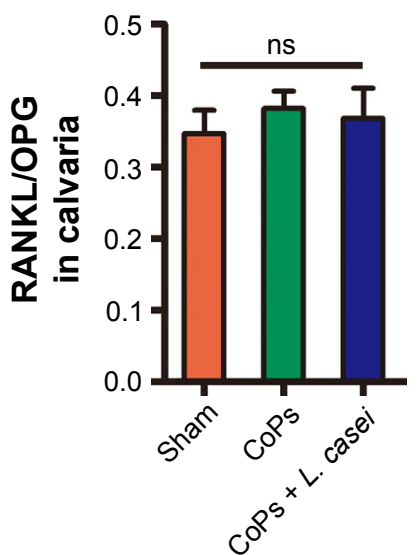

Figure 4 Lactobacillus casei did not affect RANKL and OPG expression in calvaria.

Notes: The levels of (A) RANKL, (B) OPG and (C) RANKL/OPG from each group were examined by ELISA assays. The data are presented as the mean \pm SEM ( $n=5-7$ ). Abbreviations: RANKL, receptor activator of nuclear factor (NF)-KB ligand; OPG, osteoprotegerin; ELISA, enzyme-linked immunosorbent assay; SEM, standard error of the mean; CoPs, CoCrMo particles; ns, not significant; L. casei, Lactobacillus casei. 
A
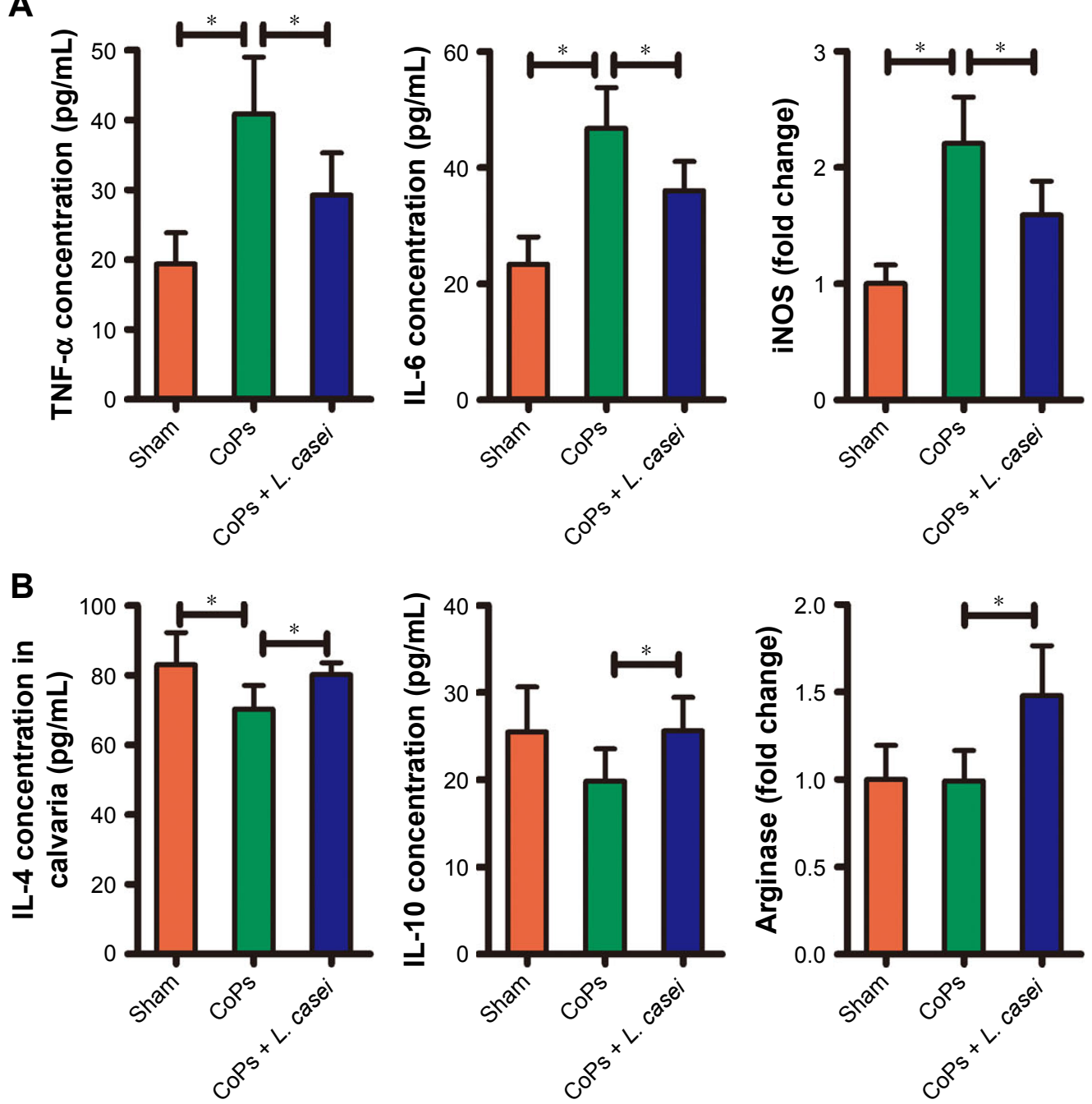

Figure 5 Lactobacillus casei decreased the levels of MI markers and enhanced the levels of M2 markers.

Notes: (A) MI polarization was inhibited in the L. casei-treated group. Expression levels of the MI signature gene (iNOS) were measured by real-time PCR, and cytokine concentrations (TNF- $\alpha$ and IL-6) in the culture supernatants were measured by ELISA. (B) M2 polarization was enhanced in the $L$. casei-treated group. Expression levels of the M2 signature gene (arginase) were measured by real-time PCR, and cytokine concentrations (IL-4 and IL-I0) in the culture supernatants were measured by ELISA. The data are presented as the mean \pm SEM $(n=5-7)$. $* P<0.05$.

Abbreviations: iNOS, inducible nitric oxide synthase; PCR, polymerase chain reaction; ELISA, enzyme-linked immunosorbent assay; TNF, tumor necrosis factor; IL, interleukin; SEM, standard error of the mean; CoPs, CoCrMo particles; L. casei, Lactobacillus casei.

responsible for the quiescent removal of apoptotic cells, participate in the regulation of tissue homeostasis and perform various tissue-specific functions. ${ }^{1,30}$ Full M1 polarization (classical macrophage activation) mediates the host's defenses against a variety of bacteria, protozoa and viruses and plays roles in antitumor immunity. Alternatively, activated macrophages (M2 macrophages) exert anti-inflammatory function and regulate wound healing. ${ }^{30}$ Wear particles can activate macrophages, which secrete proinflammatory cytokines and chemokines that lead to further macrophage recruitment, upregulation of osteoclastogenesis and downregulation of osteoblast formation and function. ${ }^{1}$ Ultimately, these changes establish a microenvironment that facilitates bone resorption over bone formation, thus causing periprosthetic osteolysis and aseptic loosening. Previous studies have suggested that M1 macrophages, rather than the M2 phenotype, predominate in the peri-implant tissue. ${ }^{1,7}$ Rao et $\mathrm{al}^{7}$ reported that the ratio of $\mathrm{M} 1 / \mathrm{M} 2$ was increased in retrieved periprosthetic tissues compared to non-operated osteoarthritic synovial tissues. In vitro studies, polymethyl methacrylate (PMMA) particles induced iNOS and TNF- $\alpha$ production in mouse bone marrow macrophages. ${ }^{7}$ In this study, L. case $i$ attenuated CoPs-induced upregulation of osteoclast formation and osteolysis in vivo. Interestingly, these effects of $L$. case $i$ were not dependent on the RANKL/OPG system. The probiotic partly reversed the CoPs-induced upregulation of TNF- $\alpha$, 
IL-6 and iNOS and the downregulation of IL-4, IL-10 and arginase, which indicated that the modulation of macrophage phenotypes may be responsible for the beneficial effects of L. casei on wear particles-induced osteolysis.

As it is well known, probiotics are nonpathogenic and have been consumed as and with food for a long time. The GM regulates bone mass, and probiotic treatment can affect the GM composition or the metabolic activity of the GM. ${ }^{8}$ Recently, various probiotics were introduced to various bone loss-related diseases and induced amazing benefits. Lactobacillus and its important species, such as L. casei and Lactobacillus acidophilus, have been used against many pathological and disease conditions. ${ }^{31}$ Lactobacillus has been assessed for its immune-modulatory properties in various experiments. ${ }^{31,32}$ In an ovariectomized (Ovx) mouse menopausal model, Britton et al found that Lactobacillus reuteri treatment significantly protected Ovx mice from bone loss. L. reuteri suppressed Ovx-induced increases in bone marrow $\mathrm{CD}^{+}{ }^{+}$-lymphocytes, which promote osteoclastogenesis, and directly suppressed osteoclastogenesis in vitro. ${ }^{33}$ In another study, the addition of $L$. reuteri prevented TNF$\alpha$-mediated suppression of Wnt10b and osteoblast maturation markers in type 1 diabetes (T1D)-induced osteoporosis. ${ }^{34}$ In this study, we showed that probiotic treatment protected mice from wear debris-induced osteolysis. Treatment with L. casei ameliorated CoPs-induced bone loss and osteoclast formation. The osteoclast markers, such as TRAP and CTR, were significantly inhibited in the CoPs plus L. casei group compared with the CoPs-treated group. Probiotic treatment reduced the expression of proinflammatory cytokines, TNF- $\alpha$ and IL- 6 and increased the expression of anti-inflammatory cytokines, IL-4 and IL-10 in the local tissue of CoPs-treated mice. These observations were consistent with previous studies in which $L$. casei treatment significantly downregulated proinflammatory cytokines, including IL-6, TNF- $\alpha$, IL-1 $\beta$ and IL-17, and upregulated anti-inflammatory cytokines, including IL-4, IL-10 and IL-13 in collagen-induced arthritic rats. ${ }^{32}$ Furthermore, we also examined the mRNA levels of M1/M2 markers. L. casei decreased the expression of the M1 marker iNOS and increased the expression of the M2 marker arginase. Thus, these data indicated that L. casei decreased the M1-like macrophage phenotype and increased the M2-like macrophage phenotype.

There are some limitations of this study. First, in addition to macrophages, other cell types (including fibroblasts, osteoblasts and other mesenchymal cells) may produce proinflammatory cytokines in response to wear particles., ${ }^{2,35}$ However, macrophages are the main cell type responsible for the expression of proinflammatory cytokines in vivo, and the changes in proinflammatory cytokines reflect the functional state of macrophages. ${ }^{2,6,15,36}$ Rao et al adopted these indexes to identify the phenotypes of macrophages. ${ }^{6}$ Second, the mechanism responsible for the change in macrophage phenotype induced by probiotics remains unclear. Most recently, we found that the expression of IL-4, a M2 macrophage inducer, was significantly upregulated in serum in the $L$. casei-treated group (data not shown). Further investigations on the expression of inducers of M1 and M2 polarization in serum and the interaction between probiotics and intestinal epithelial cells in vitro may facilitate our understanding of the benefits of probiotics.

\section{Conclusion}

This study demonstrated that treatment with L. case $i$ attenuated CoPs-induced osteolysis in vivo. Our findings indicated that the probiotic treatments may alter the immune status in local tissue, as demonstrated by the reduced M1-like macrophage phenotype and the increased M2-like macrophage phenotype, resulting in the amelioration of bone loss in mouse calvarial models. These results suggested a preventive and therapeutic potential of probiotics in the treatment of this disease.

\section{Acknowledgments}

This work was supported by the National Natural Science Foundation of China (31200695 and 81572111), the Natural Science Foundation of Jiangsu Province (BK20161385), the Department of Orthopedics Clinical Research Center of Jiangsu Province, People's Republic of China (BL2012002), the Science and Technology Project of Nanjing (201402007) and the Scientific Research Foundation of Graduate School of Nanjing University (2015CL12). The authors thank Dr L Guo for his generous assistance in obtaining and culture of human specimens.

\section{Disclosure}

The authors report no conflicts of interest in this work.

\section{References}

1. Goodman SB, Gibon E, Pajarinen J, et al. Novel biological strategies for treatment of wear particle-induced periprosthetic osteolysis of orthopaedic implants for joint replacement. J R Soc Interface. 2014;11(93): 20130962.

2. Wang R, Wang Z, Ma Y, et al. Particle-induced osteolysis mediated by endoplasmic reticulum stress in prosthesis loosening. Biomaterials. 2013; 34(11):2611-2623.

3. Prokopetz JJZ, Losina E, Bliss RL, Wright J, Baron JA, Katz JN. Risk factors for revision of primary total hip arthroplasty: a systematic review. BMC Musculoskelet Disord. 2012;13:251. 
4. Wooley PH, Schwarz EM. Aseptic loosening. Gene Ther. 2004;11(4): 402-407.

5. Gallo J, Goodman SB, Konttinen YT, Raska M. Particle disease: biologic mechanisms of periprosthetic osteolysis in total hip arthroplasty. Innate Immun. 2013;19(2):213-224.

6. Rao AJ, Nich C, Dhulipala LS, et al. Local effect of IL-4 delivery on polyethylene particle induced osteolysis in the murine calvarium. J Biomed Mater Res A. 2013;101(7):1926-1934.

7. Rao AJ, Gibon E, Ma T, Yao Z, Smith RL, Goodman SB. Revision joint replacement, wear particles, and macrophage polarization. Acta Biomater. 2012;8(7):2815-2823.

8. Ohlsson C, Engdahl C, Fak F, et al. Probiotics protect mice from ovariectomy-induced cortical bone loss. PLoS One. 2014;9(3):e92368.

9. Sjogren K, Engdahl C, Henning P, et al. The gut microbiota regulates bone mass in mice. $J$ Bone Miner Res. 2012;27(6):1357-1367.

10. Vaghef-Mehrabany E, Alipour B, Homayouni-Rad A, Sharif SK, Asghari-Jafarabadi M, Zavvari S. Probiotic supplementation improves inflammatory status in patients with rheumatoid arthritis. Nutrition. 2014;30(4):430-435.

11. Kato I, Endo-Tanaka K, Yokokura T. Suppressive effects of the oral administration of Lactobacillus casei on type II collagen-induced arthritis in DBA/1 mice. Life Sci. 1998;63(8):635-644.

12. So JS, Lee CG, Kwon HK, et al. Lactobacillus casei potentiates induction of oral tolerance in experimental arthritis. Mol Immunol. 2008;46(1): $172-180$.

13. Amdekar S, Singh V, Singh R, Sharma P, Keshav P, Kumar A. Lactobacillus casei reduces the inflammatory joint damage associated with collagen-induced arthritis (CIA) by reducing the pro-inflammatory cytokines: Lactobacillus casei: COX-2 inhibitor. J Clin Immunol. 2011 31(2):147-154.

14. Rieu A, Aoudia N, Jego G, et al. The biofilm mode of life boosts the anti-inflammatory properties of Lactobacillus. Cell Microbiol. 2014; 16(12):1836-1853

15. Nich C, Takakubo Y, Pajarinen J, et al. Macrophages-key cells in the response to wear debris from joint replacements. J Biomed Mater Res A. 2013;101(10):3033-3045.

16. Frellsen AF, Hansen AE, Jolck RI, et al. Mouse positron emission tomography study of the biodistribution of gold nanoparticles with different surface coatings using embedded copper-64. ACS Nano. 2016 10(11):9887-9898.

17. Wang Z, Deng Z, Gan J, et al. TiAl6V4 particles promote osteoclast formation via autophagy-mediated downregulation of interferon-beta in osteocytes. Acta Biomater. 2017;48:489-498.

18. Wang Z, Liu N, Liu K, et al. Autophagy mediated CoCrMo particleinduced peri-implant osteolysis by promoting osteoblast apoptosis. Autophagy. 2015;11(12):2358-2369.

19. Wang Z, Liu N, Shi T, et al. ER stress mediates TiAl6V4 particleinduced peri-implant osteolysis by promoting RANKL expression in fibroblasts. PLoS One. 2015;10(9):e0137774.

20. Liu X, Qu X, Wu C, et al. The effect of enoxacin on osteoclastogenesis and reduction of titanium particle-induced osteolysis via suppression of JNK signaling pathway. Biomaterials. 2014;35(22):5721-5730.
21. Brown C, Williams S, Tipper JL, Fisher J, Ingham E. Characterisation of wear particles produced by metal on metal and ceramic on metal hip prostheses under standard and microseparation simulation. J Mater Sci Mater Med. 2007;18(5):819-827.

22. Catelas I, Bobyn JD, Medley JB, Krygier JJ, Zukor DJ, Huk OL. Size, shape, and composition of wear particles from metal-metal hip simulator testing: effects of alloy and number of loading cycles. J Biomed Mater Res A. 2003;67(1):312-327.

23. Milosev I, Remskar M. In vivo production of nanosized metal wear debris formed by tribochemical reaction as confirmed by high-resolution TEM and XPS analyses. J Biomed Mater Res A. 2009;91(4):1100-1110.

24. Firkins PJ, Tipper JL, Saadatzadeh MR, et al. Quantitative analysis of wear and wear debris from metal-on-metal hip prostheses tested in a physiological hip joint simulator. Biomed Mater Eng. 2001;11(2): $143-157$.

25. Nine M, Choudhury D, Hee A, Mootanah R, Osman N. Wear debris characterization and corresponding biological response: artificial hip and knee joints. Materials. 2014;7(2):980-1016.

26. Choi MG, Koh HS, Kluess D, et al. Effects of titanium particle size on osteoblast functions in vitro and in vivo. Proc Natl Acad Sci U S A. 2005;102(12):4578-4583.

27. O'Neill SC, Queally JM, Devitt BM, Doran PP, O'Byrne JM. The role of osteoblasts in peri-prosthetic osteolysis. Bone Joint J. 2013;95-B(8): 1022-1026.

28. Boyle WJ, Simonet WS, Lacey DL. Osteoclast differentiation and activation. Nature. 2003;423(6937):337-342.

29. Mantovani A, Sozzani S, Locati M, Allavena P, Sica A. Macrophage polarization: tumor-associated macrophages as a paradigm for polarized M2 mononuclear phagocytes. Trends Immunol. 2002;23(11): 549-555.

30. Murray PJ, Wynn TA. Protective and pathogenic functions of macrophage subsets. Nat Rev Immunol. 2011;11(11):723-737.

31. Amdekar S, Roy P, Singh V, Kumar A, Singh R, Sharma P. Antiinflammatory activity of Lactobacillus on carrageenan-induced paw edema in male wistar rats. Int J Inflamm. 2012;2012:752015.

32. Amdekar S, Singh V, Kumar A, Sharma P, Singh R. Lactobacillus casei and Lactobacillus acidophilus regulate inflammatory pathway and improve antioxidant status in collagen-induced arthritic rats. J Interferon Cytokine Res. 2013;33(1):1-8.

33. Britton RA, Irwin R, Quach D, et al. Probiotic L. reuteri treatment prevents bone loss in a menopausal ovariectomized mouse model. J Cell Physiol. 2014;229(11):1822-1830.

34. Zhang J, Motyl KJ, Irwin R, MacDougald OA, Britton RA, McCabe LR. Loss of bone and Wnt $10 \mathrm{~b}$ expression in male type 1 diabetic mice is blocked by the probiotic Lactobacillus reuteri. Endocrinology. 2015; 156(9):3169-3182.

35. Gallo J, Raska M, Mrazek F, Petrek M. Bone remodeling, particle disease and individual susceptibility to periprosthetic osteolysis. Physiol Res. 2008;57(3):339-349.

36. Nich C, Goodman SB. Role of macrophages in the biological reaction to wear debris from joint replacements. $J$ Long Term Eff Med Implants. 2014;24(4):259-265. 


\section{Supplementary materials}
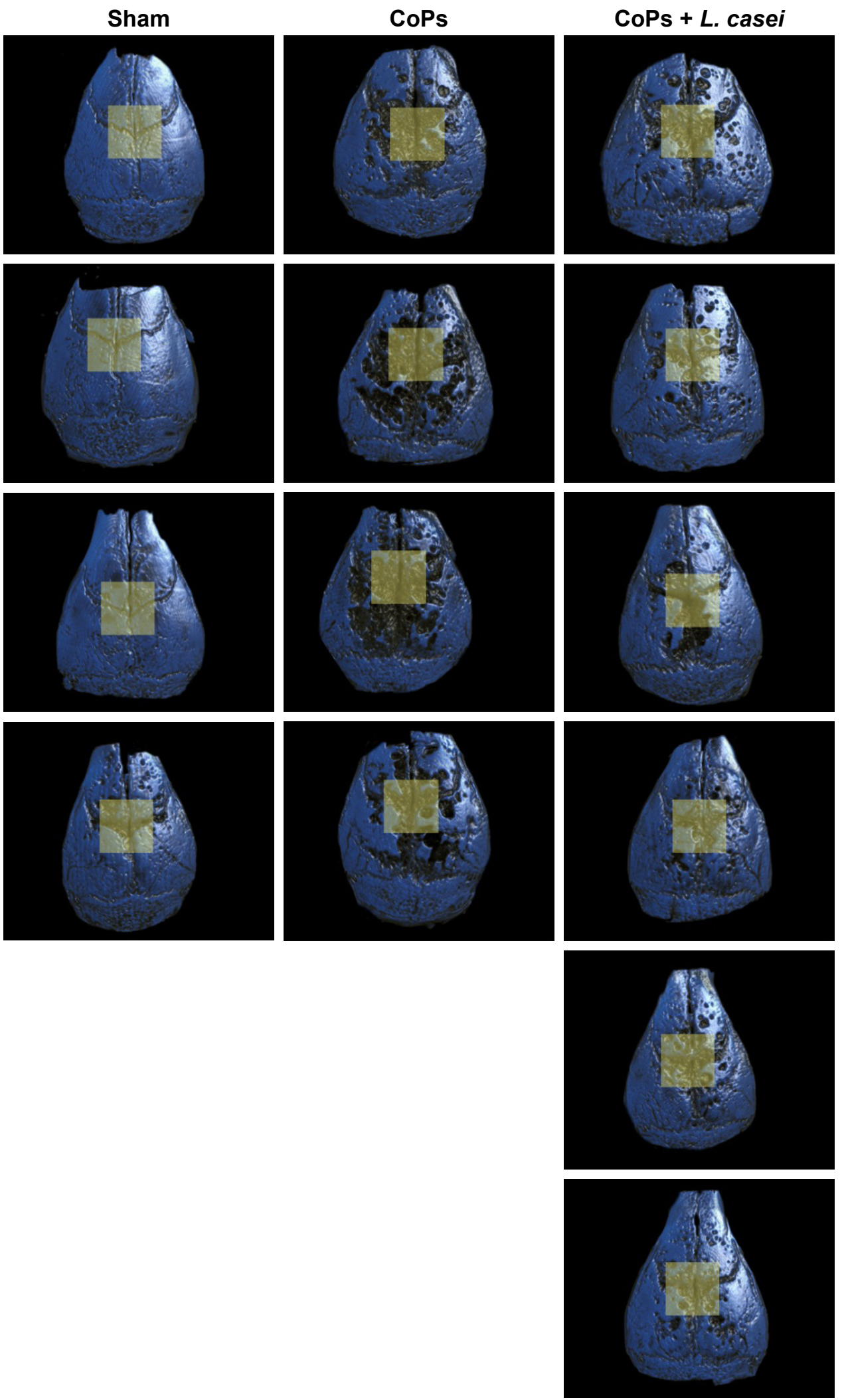

Figure SI Lactobacillus casei ameliorated CoPs-induced mouse calvarial osteolysis.

Notes: Micro-CT with three-dimensional reconstructed images from each group. The ROI was indicated by the yellow square box.

Abbreviations: CoPs, CoCrMo particles; CT, computed tomography; ROI, region of interest; L. casei, Lactobacillus casei. 

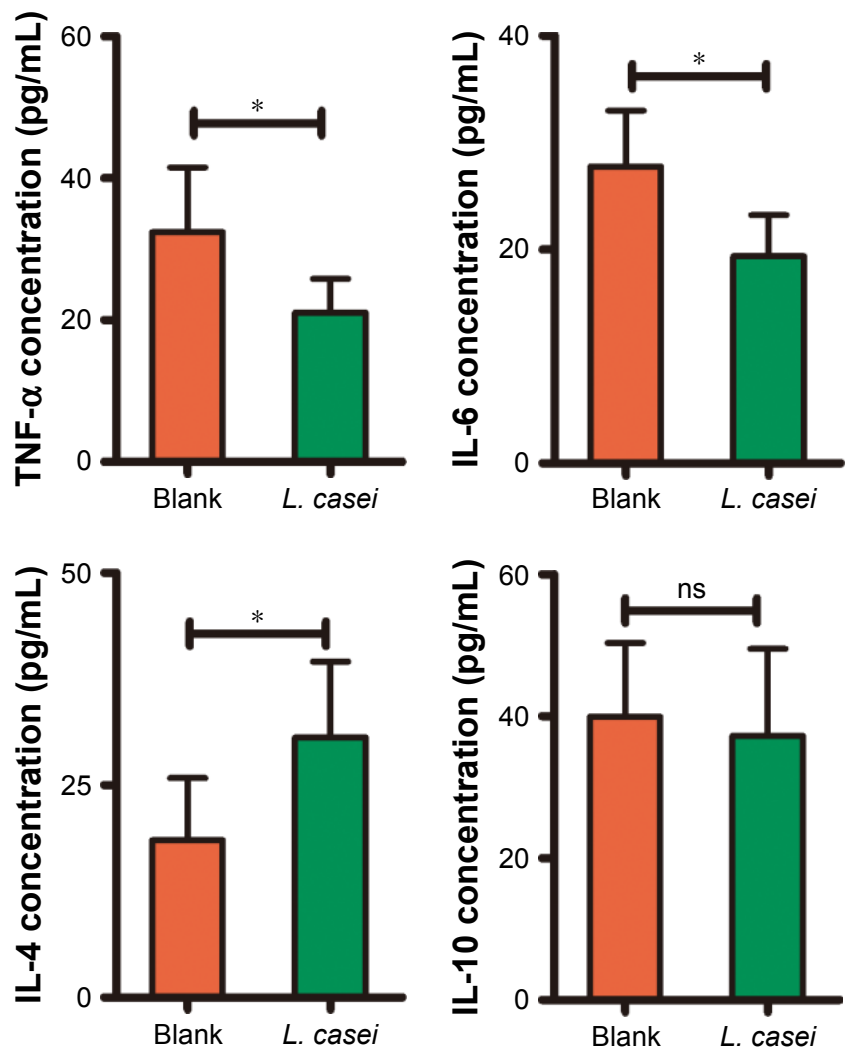

Figure S2 The effect of Lactobacillus casei on the levels of TNF- $\alpha$, IL-6, IL-4 and IL- 10 in vitro.

Notes: The levels of TNF- $\alpha$, IL-6, IL- 4 and IL- 10 in the incubation medium after 24 hours culture of colonic mucosa. The data are presented as the mean \pm SEM. $* P<0.05$. Abbreviations: TNF, tumor necrosis factor; IL, interleukin; SEM, standard error of the mean; L. casei, Lactobacillus casei; ns, not significant.

\section{Publish your work in this journal}

The International Journal of Nanomedicine is an international, peerreviewed journal focusing on the application of nanotechnology in diagnostics, therapeutics, and drug delivery systems throughout the biomedical field. This journal is indexed on PubMed Central, MedLine, CAS, SciSearch ${ }^{\circledR}$, Current Contents ${ }^{\circledR} /$ Clinical Medicine,
Journal Citation Reports/Science Edition, EMBase, Scopus and the Elsevier Bibliographic databases. The manuscript management system is completely online and includes a very quick and fair peer-review system, which is all easy to use. Visit http://www.dovepress.com/ testimonials.php to read real quotes from published authors 